\title{
ABL1 Is a Prognostic Marker and Associated with Immune Infiltration in Hepatocellular Carcinoma
}

\author{
Rongqiang Liu $\mathbb{D}^{1,2}$ Weihao Kong $\mathbb{D}^{1,2}$ ZeKun Jiang $\mathbb{D}^{4},{ }^{4}$ Shiyang Zheng $\mathbb{D}^{5}{ }^{5}$ \\ Xiaofeng Yuan ${ }^{D},{ }^{6}$ and Linsen Ye ${ }^{1}$ \\ ${ }^{1}$ Department of Hepatic Surgery and Liver Transplantation Center, The Third Affiliated Hospital of Sun Yat-Sen University, \\ Guangzhou 510630, China \\ ${ }^{2}$ Department of Hepatobiliary Surgery, The First Affiliated Hospital of Guangzhou Medical University, Guangzhou 510220, \\ Guangdong, China \\ ${ }^{3}$ Department of Emergency Surgery, The First Affiliated Hospital of Anhui Medical University, Hefei, Anhui 230022, China \\ ${ }^{4}$ Department of Gastrointestinal Surgery, The First Affiliated Hospital of Guangzhou Medical University, Guangzhou 510220, \\ Guangdong, China \\ ${ }^{5}$ Department of Breast Surgery, The Third Affiliated Hospital of Guangzhou Medical University, Guangzhou 510150, China \\ ${ }^{6}$ Department of General ICU, The Third Affiliated Hospital of Sun Yat-Sen University-LingNan Hospital, \\ Guangzhou 510630, China
}

Correspondence should be addressed to Xiaofeng Yuan; yuanxf5@mail.sysu.edu.cn and Linsen Ye; ye_linsen@163.com

Rongqiang Liu, Weihao Kong, and ZeKun Jiang contributed equally to this work.

Received 8 April 2021; Revised 2 August 2021; Accepted 6 August 2021; Published 26 August 2021

Academic Editor: Alessandro Granito

Copyright (c) 2021 Rongqiang Liu et al. This is an open access article distributed under the Creative Commons Attribution License, which permits unrestricted use, distribution, and reproduction in any medium, provided the original work is properly cited.

Background. The role of ABL1 in hepatocellular carcinoma (HCC) is still unclear. Therefore, this study aims to explore the potential role of ABL1 in the progression of HCC using bioinformatics methods. Methods. We analyzed the expression, prognostic potential, and immune cell effect of ABL1 in HCC by using a variety of datasets. Results. ABL1 is highly expressed in HCC and associated with unfavorable overall survival (OS) and disease-free survival (DFS). Functional network analysis revealed that ABL1 plays an important role in mitochondrial activity, ATP metabolism, protein translation and metabolism, various neurological diseases, nonalcoholic fatty liver disease, and notch signaling pathway. In addition, we found that ABL1 expression was closely correlated with B cells, CD8 + T cells, CD4 + T cells, macrophages, neutrophils, and dendritic cells. Furthermore, ABL1 expression was positively associated with the expression levels of immune checkpoint genes, such as PD-1L, TIM3, TIGIT, and CTLA4. Conclusion. ABL1 is associated with immune infiltration and prognosis of HCC.

\section{Introduction}

Hepatocellular carcinoma (HCC) is the most common primary liver tumor and the second most common risk factor for cancer death worldwide [1]. In China, approximately 400,000 people die from liver cancer each year, accounting for more than $50 \%$ of the global liver cancer death rate [2]. According to statistics, the incidence of liver cancer will continue to rise in the next 20 years [3]. HCC prevention and treatment have become an urgent problem for the global medical community. In its early stages, HCC is occult and difficult to detect; thus, patients with
HCC are often diagnosed when the cancer has already advanced or metastasized. Although great progress has been made in the comprehensive clinical treatment of HCC, the prognosis of patients with advanced liver cancer is still poor [4]. Therefore, finding new and specific tumor markers to effectively predict the prognosis of HCC patients is of great value for guiding clinical treatment and improving patient survival.

The human Abelson tyrosine-protein kinase 1 (ABL1) gene is located on chromosome $9 \mathrm{q} 34$, and the ABL1 protein encoded by it belongs to the ABL interacting protein family [5]. ABL1 plays a role in a wide range of normal biological 
functions, including cytoskeleton remodeling [6]. In 1998, Taki et al. revealed that ABL1 was associated with the occurrence of chronic myeloid leukemia [5]. Since then, knowledge of the roles of ABL1 has gradually increased. Subsequent studies have shown that ABL1 is abnormally expressed in a variety of tumors and plays a crucial role in tumor proliferation, migration, invasion, and metastasis [7]. ABL1 regulates a variety of signaling pathways, such as the EGF and PI3K/AKT signaling pathways $[8,9]$. In addition, ABL1 has been found to be closely associated with the prognosis of some tumors, including gastric and breast cancers $[10,11]$.

So far, only a few studies have reported the relationship between ABL1 and HCC. Chitsike et al. indicated that ABL1 was abnormally expressed in human liver cancer [12]. Wang et al. further found that overexpression of ABL1 promoted tumor progression through NOTCH1 in mouse liver cancer [13]. However, the prognosis and clinical value of ABL1 in HCC in humans is still unclear.

Immune infiltration is known to be closely related to the progression of liver cancer [14]. In this study, we used a variety of databases to comprehensively explore the clinical significance of ABL1 in liver cancer, identify possible target pathways, and determine the relationship between ABL1 and immune infiltration. Our results provide a better understanding of the potential value of ABL1 in liver cancer.

\section{Materials and Methods}

2.1. Data Acquisition. Datasets with gene expression profiles and clinical information regarding HCC mRNA expression were downloaded from the TCGA database [15] (https:// TCGA data.nci.nih.gov/tcga/). Standardization was performed on the downloaded HCC datasets, and cases that did not contain survival information were excluded. The total number of samples in the original study of the TCGA data was 529, consisting of 369 liver cancer tissue samples and 160 paracancerous tissue samples. Five HCC samples contained no survival information. Using the median of ABL1 mRNA expression as a node, HCC tissue samples with survival information were divided according to ABL1 expression levels into a high expression group and a low expression group, with 182 samples in each group.

2.2. Survival and Expression Analyses. The online database Gene Expression Profiling Interactive Analysis (GEPIA) [16] was used to analyze the expression of ABL1 in HCC tissues and normal tissues and further assess the prognostic value of ABL1 in liver cancer. In addition, we conducted univariate or multivariate analysis on the clinicopathological information available on ABL1 in HCC. Moreover, we used the online database UALCAN [17] to evaluate the expression of ABL1 protein in different groups.

2.3. Functional Enrichment Analysis. The LinkedOmics database [18] is a free public website that can analyze TCGA data. The LinkedOmics database was used to analyze ABL1related genes. The Pearson correlation coefficient was applied for statistical analysis, and the results are expressed as a volcano map and a heat map. In addition, Gene Ontology Biological Process (GOBP) and Kyoto Encyclopedia of Genes and Genomes (KEGG) pathway analyses were performed. Genes with a discovery rate $(\mathrm{FDR})<0.05$ were considered to be significantly enriched.

2.4. TIMER Database Analysis. TIMER [19] is a visualization website that can perform automatic analysis and correlate the immune penetration level and immunogenicity. The TIMER website uses deconvolution to analyze gene expression profiles to infer the expression of tumor-infiltrating immune cells. We used the TIMER database to explore the relationship between the expression abundance of various immune cells and the expression of ABL1 in HCC.

\subsection{Evaluation of Tumor Microenvironment via CIBERSORT.} CIBERSORT [20] can assess changes in the expression of specific genes in tissue based on a deconvolution algorithm. We used CIBERSORT to evaluate the immune response of 22 immune cell types in HCC. The "Vioplot" package was used to visualize the differences in the 22 TIICs between the high and low ABL1 expression groups. In addition, TISIDB [21] was used to analyze the relationship between ABL1 expression and 28 types of tumorinfiltrating lymphocytes.

2.6. Statistical Analysis. The statistical data obtained from TCGA were processed by $R 3.5 .3$, and the $P$ value $<0.05$ was considered statistically significant. The survival rate was analyzed by a log-rank test and Mantel-Cox test. Logistic regression was used to analyze the correlation between clinical features and ABL1 expression. The correlation between the expression of ABL1, coexpressed genes, and 22 immune cell types was measured by the Pearson correlation coefficient.

\section{Results}

3.1. Expression Levels and Prognostic Value of ABL1 in HCC. To evaluate the expression of ABL1 in HCC tissues and normal tissues, we used GEPIA to analyze 369 HCC specimens from TCGA. This analysis revealed that ABL1 expression was significantly higher in HCC tissues than in normal tissues (Figure 1). In addition, we performed a subgroup analysis based on age, sex, cancer stage, and TP53 mutation status (Figure 2) and found that ABL1 expression was significantly higher in HCC patients than in healthy people.

In addition, we analyzed the association between ABL1 expression and clinicopathological characteristics in the TCGA HCC cohort (Table 1). ABL1 expression was significantly correlated with gender $(<0.01)$, histological grade $(P=0.013)$, and survival status $(P<0.01)$. We further analyzed the prognostic value of ABL1 in HCC. The survival curve from GEPIA showed that high expression of ABL1 predicts unfavorable OS $(P=0.014)$ and DFS $(P=0.035)$. 


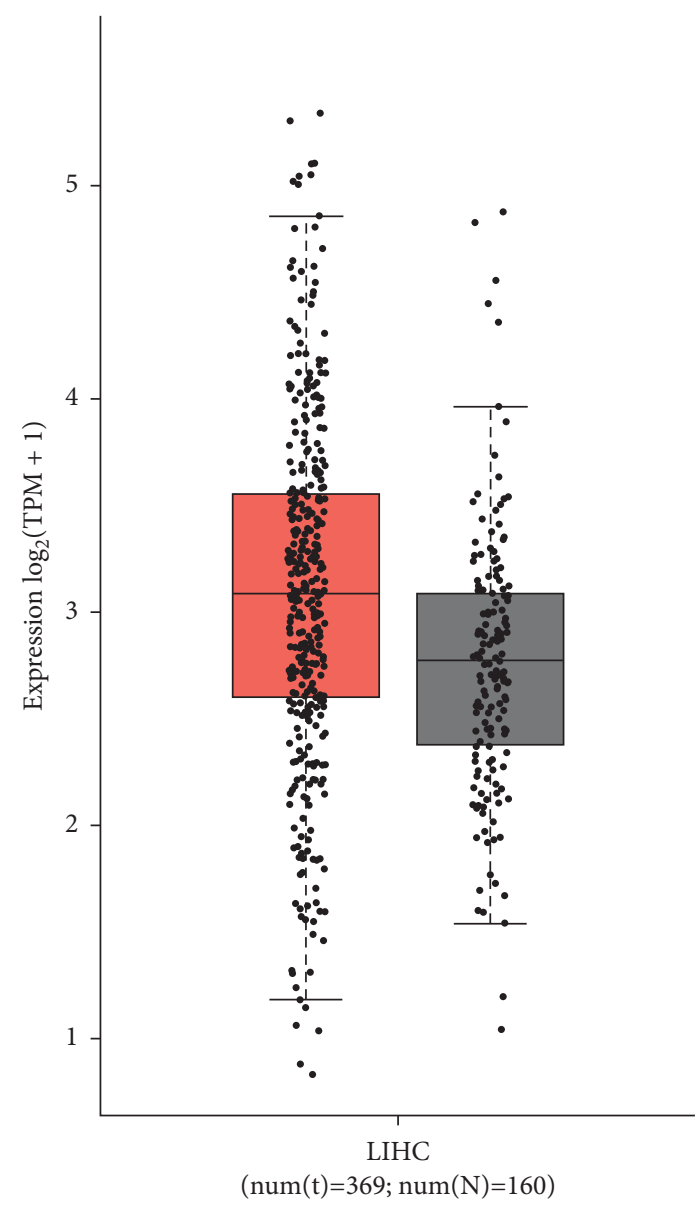

Figure 1: ABL1 mRNA expression in HCC. ABL1 mRNA expression in liver cancer tissues is significantly higher than that in normal tissues.

The results are shown in Figure 3. We also explored the relationship between various clinicopathological features, ABL1 expression, and prognosis of HCC. The results of both univariate and multivariate analyses indicated that ABL1 expression was associated with the prognosis of HCC (Figure 4) (Table 2). These results suggest that ABL1 is a potentially effective independent prognostic marker for HCC.

3.2. ABL1-Related Functions and Pathways in HCC. We explored the biological interaction network of ABL1 in HCC to clarify the biological function of ABL1. We first selected the genes related to ABL1 and performed an enrichment analysis. The top 50 genes with significant positive and negative correlations with ABL1 are shown in Figures 5(a) and 5(b). In addition, we performed GO and KEGG analyses. GO analysis showed that these genes mainly regulate mitochondrial activity, ATP metabolism, protein translation, and metabolism (Figure 5(c)). KEGG pathway analysis showed enrichment in various neurological diseases, nonalcoholic fatty liver disease, and the notch signaling pathway (Figure 5(d)). These findings indicated the potential role of ABL1 in HCC progression.
3.3. Association between ABL1 Expression and Tumor-Infiltrating Immune Cells. Immune infiltration is closely related to tumor progression. Therefore, we also evaluated the effect of ABL1 expression on immune infiltrating cells in liver cancer using CIBERSORT. The proportions of 22 immune cell subgroups are shown in Figure 6. The results showed that $\mathrm{B}$ cells, dendritic cells, macrophages, mast cells, monocytes, NK cells, CD4 cells, and CD8 cells are significantly affected by ABL1 expression. Among the identified cells, naive $\mathrm{B}$ cells $(P=0.001623), \quad$ M2 macrophages $(P=0.003429)$, mast cells resting $(P=6.74 e-7)$, and NK cells resting $(P=0.0002681)$ were most abundant in the high ABL1 expression group, whereas dendritic cells resting $(P=4.747 e-05), \mathrm{M} 0$ macrophages $(P=3.308 e-12), \mathrm{CD} 4$ cells $(P=0.0008109)$, and CD8 cells $(P=0.01354)$ were significantly reduced.

We used TIMER to further study the association between ABL1 and the level of tumor immune cell infiltration (Figure 7(a)). The results revealed that high ABL1 expression was significantly positively correlated with B cells $(r=0.285$, $P=7.25 e-08)$, CD8 cells $(r=0.212, P=7.68 e-06)$, CD 4 cells $(r=0.496, P=8.43 e-23)$, macrophages $(r=0.46$, $P=3.24 e-19)$, neutrophils $(r=0.488, P=4.32 e-22)$, and dendritic cells $(r=0.394, P=4.35 e-14)$ in HCC. These results suggest that ABL1 expression influences liver cancer progression by altering immune cell infiltration.

3.4. Association between ABL1 Expression and Immune Checkpoints. Immune checkpoint blockade therapy is a popular immunotherapy method and shows a strong therapeutic effect. We explored the relationship between ABL1 expression and tumor immunotherapy. We found that some immune checkpoints (PD-1L, TIM3, TIGIT, and CTLA4) were positively correlated with ABL1 expression (Figure 7(b)). In addition, these immune checkpoint markers were significantly expressed in the group with high ABL1 expression. We further explored the relationship between ABL1 and various infiltrating immune cell types. The results showed that the expression level of ABL1 was positively correlated with $\mathrm{T}$ cell exhaustion, $\mathrm{T}$ cells (general), CD8 + T cells, CD4 + T cells, Th1 cells, Th2 cells, Tfh cells, Th17 cells, Tregs, monocytes, TAMs, M1 macrophages, M2 macrophages, neutrophils, natural killer cells, and dendritic cells. The results are shown in Table 3. This finding suggests that ABL1 is involved in T cell exhaustion in the hepatocellular carcinoma tumor microenvironment.

\section{Discussion}

HCC can occur at any age and is most common in patients with chronic hepatitis [22]. Viral hepatitis B is highly prevalent in China, and HCC incidence shows an increasing trend year by year [23]. HCC is highly malignant, and surgical resection is currently the only curative therapeutic intervention. Although great progress has been made in developing diagnostic tools and treatments for liver cancer, the prognosis of most patients with advanced HCC is still very unsatisfactory. ABL1 is a gene that was first reported in 


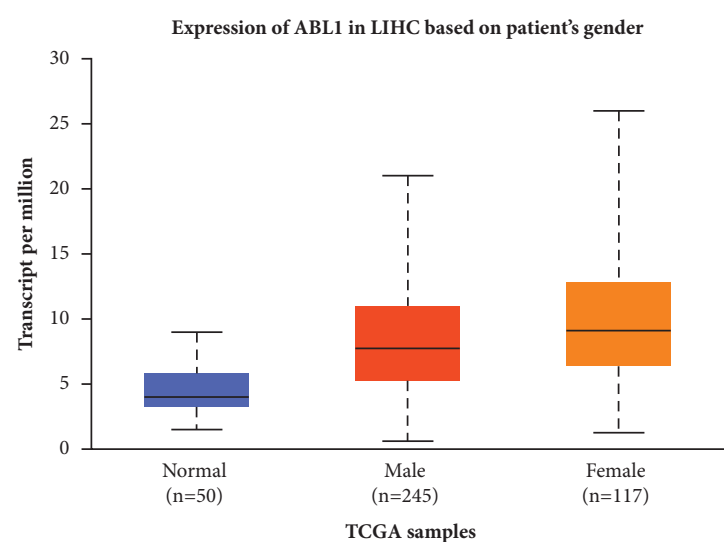

(a)

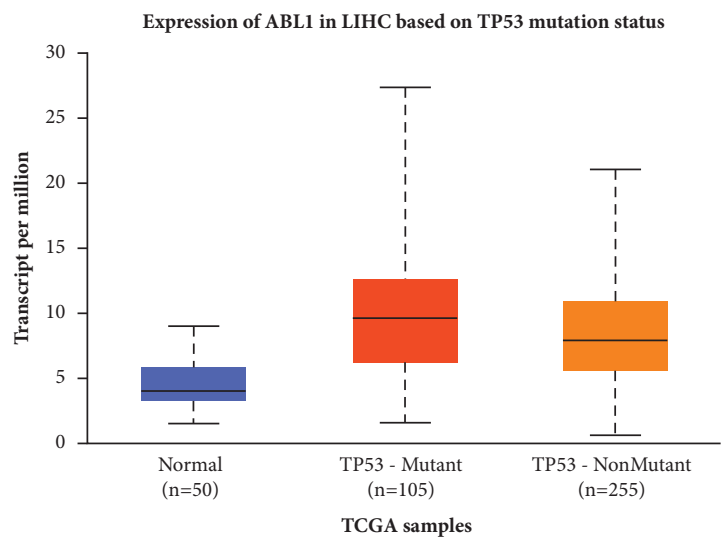

(c)

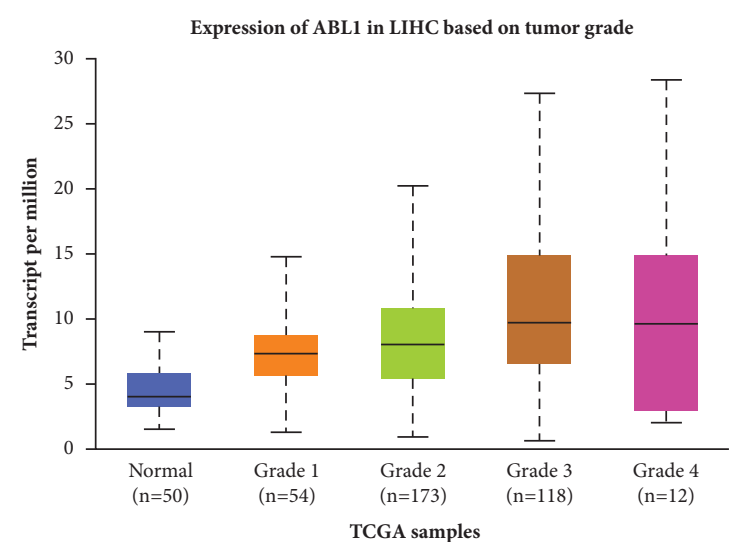

(b)

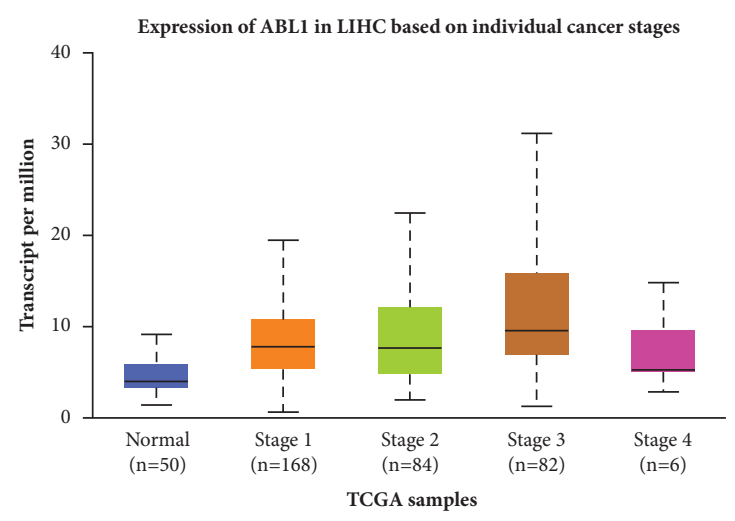

(d)

FiguRE 2: ABL1 protein expression varies in various subgroups of HCC patients based on patient age, sex, cancer stage, and TP53 mutation status. (a) Box plot shows the relative expression of ABL1 in different age groups of HCC patients. (b) Box plot shows the relative expression of ABL1 in different sex groups of HCC patients. (c) Box plot shows the relative expression of ABL1 in the cancer stage group of HCC patients. (d) Box plot shows the relative expression of ABL1 in the TP53 mutation status group of HCC patients.

TABLe 1: Association between ABL1 expression and clinicopathological characteristics in the TCGA HCC cohort.

\begin{tabular}{|c|c|c|c|c|}
\hline Variable & Low ABL1 expression $(n=170)$ & High ABL1 expression $(n=171)$ & $X^{2}$ & $P$ value \\
\hline Age & & & 0.8026 & 0.3703 \\
\hline$\leq 50$ & 33 & 40 & & \\
\hline$>50$ & 137 & 131 & & \\
\hline Gender & & & 6.6817 & $<0.01$ \\
\hline Female & 49 & 80 & & \\
\hline Male & 121 & 111 & & \\
\hline TNM stage & & & 3.1116 & 0.119 \\
\hline $\mathrm{I} / \mathrm{II}$ & 124 & 114 & & \\
\hline III/IV & 46 & 57 & & \\
\hline Histologic grade & & & 6.1729 & 0.013 \\
\hline $\mathrm{G} 1 / \mathrm{G} 2$ & 123 & 91 & & \\
\hline G3/G4 & 47 & 80 & & \\
\hline \multicolumn{5}{|l|}{ Survival status } \\
\hline Alive & 113 & 105 & 48.0403 & $<0.01$ \\
\hline Dead & 57 & 66 & & \\
\hline
\end{tabular}

leukemia. Subsequently, abnormal ABL1 expression was found in a variety of other tumors. However, there are few studies on the prognostic value and specific mechanism of
ABL1 in liver cancer. Therefore, our research focused on the potential relationship between ABL1 and HCC. We aimed to evaluate the specific biological function of ABL1 in HCC 


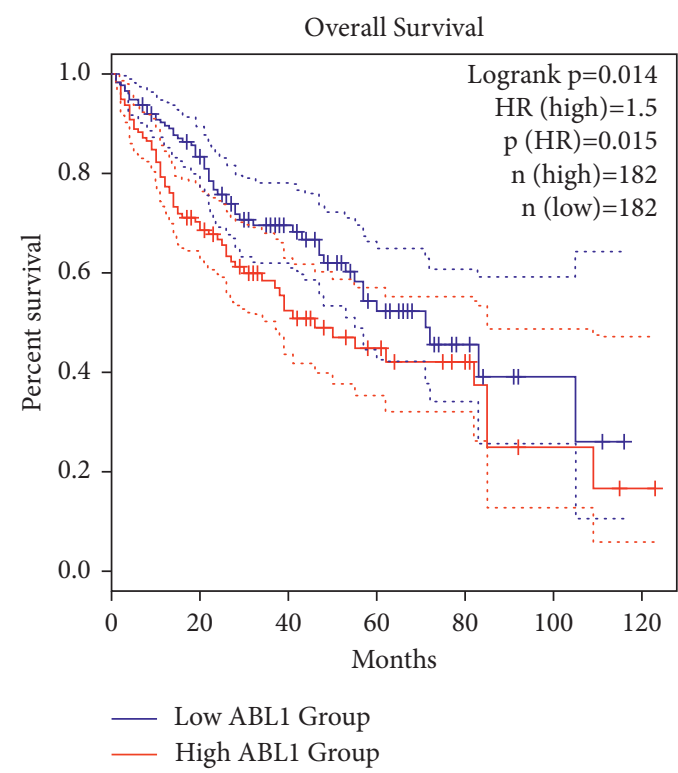

(a)

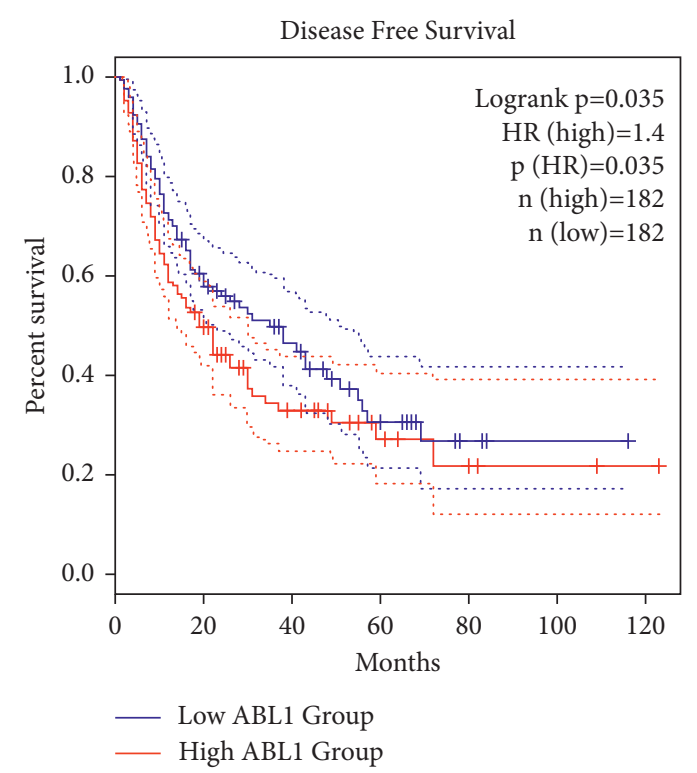

(b)

FIgure 3: The prognostic value of ABL1 in HCC. (a) OS. (b) DFS.

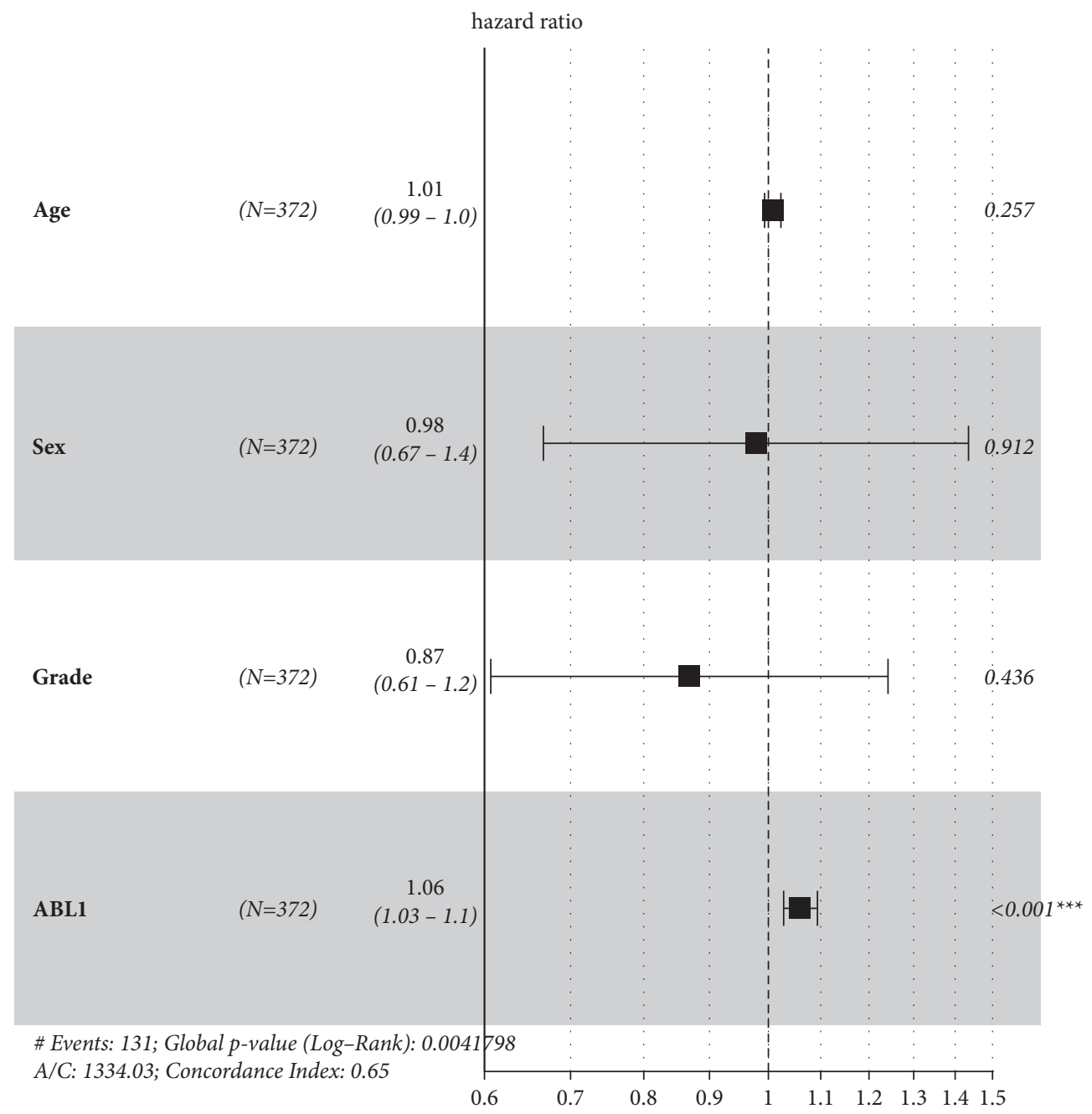

Figure 4: Multivariate analysis of ABL1 expression and other clinical characteristics. 
TABLE 2: Univariate and multivariate Cox regression of ABL1 expression for overall survival in patients with HCC.

\begin{tabular}{lcccc}
\hline Variable & Univariate Cox regression HR $(95 \% \mathrm{CI})$ & $P$ value & Multivariate Cox regression HR (95\% CI) & $P$ value \\
\hline Age & $1.008(0.995-1.022)$ & 0.221 & $1.0083(0.9940-1.023)$ & 0.257303 \\
Sex & $0.8039(0.5647-1.144)$ & 0.226 & $0.9787(0.6671-1.436)$ & 0.912241 \\
Grade & $0.8703(0.6133-1.235)$ & 0.437 & $0.8675(0.6068-1.240)$ & 0.435503 \\
ABL1 & $1.062(1.032-1.093)$ & $4.61 E-05$ & $1.0597(1.0286-1.092)$ & 0.000138 \\
\hline
\end{tabular}

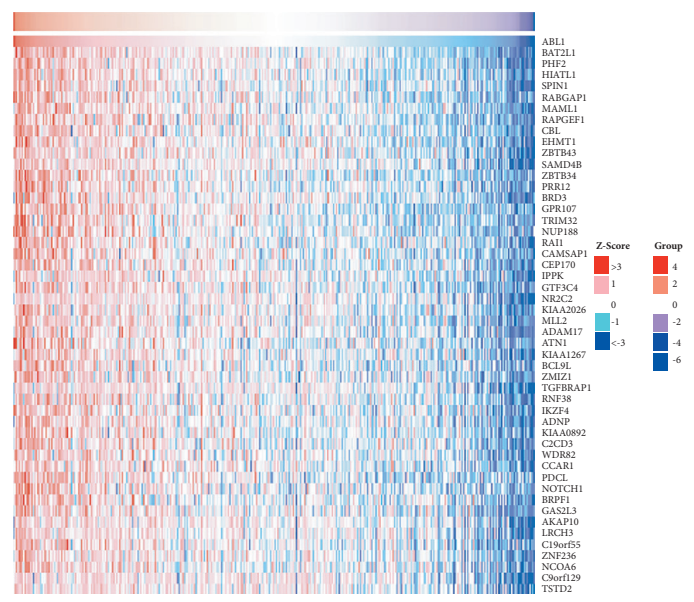

(a)

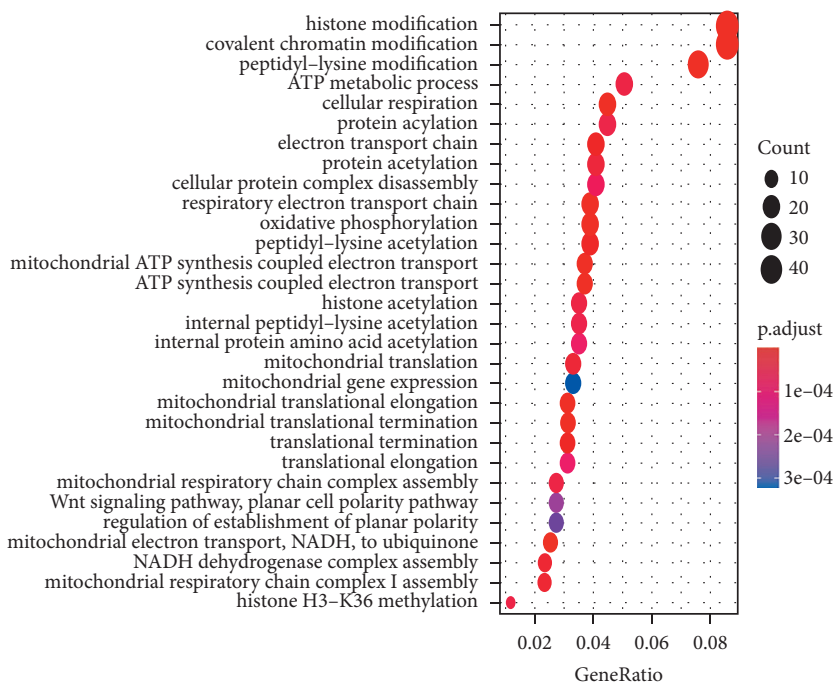

(c)

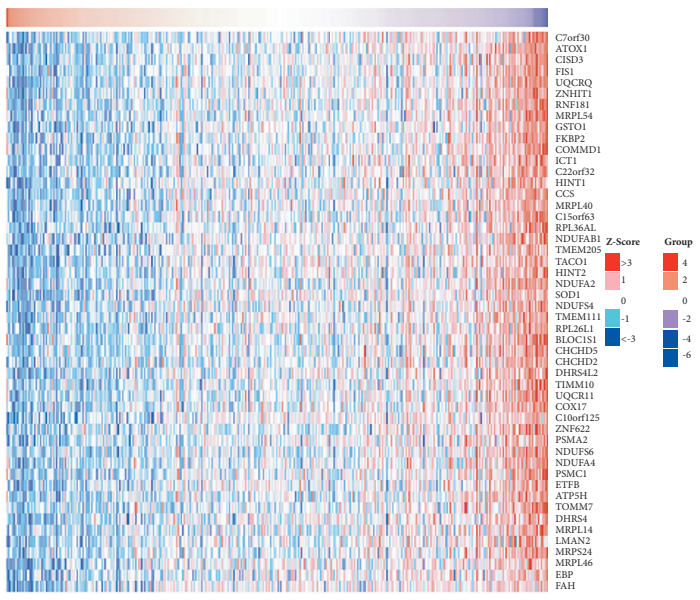

(b)

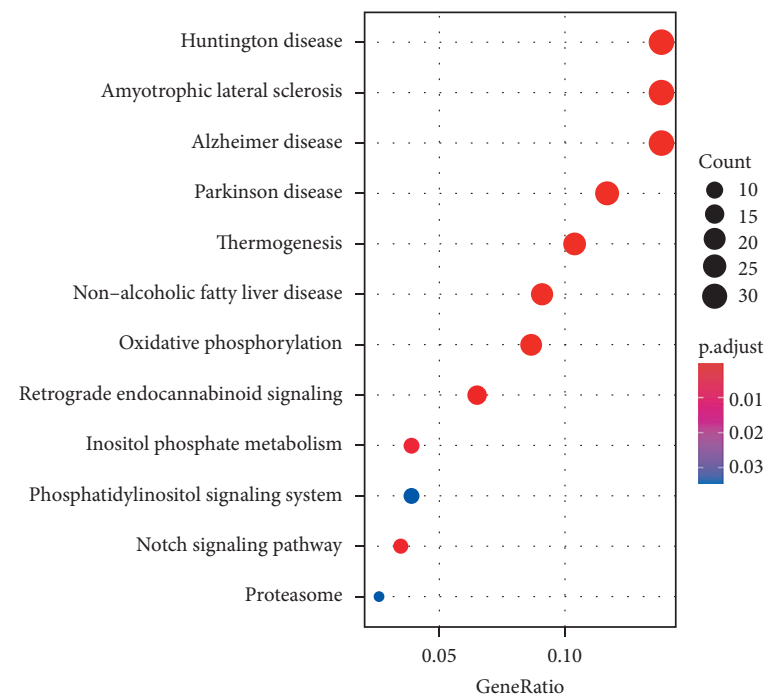

(d)

FIgURE 5: ABL1 coexpressed genes and functional enrichment. (a) The heat map shows the top 50 genes positively related to ABL1. (b) The heat map shows the top 50 genes negatively related to ABL1. (c) GO enrichment analysis. (d) KEGG enrichment analysis.

through bioinformatics methods to identify ABL1-related pathways and determine the association between ABL1 and tumor immunity.

Our analysis showed that ABL1 expression was significantly higher in liver cancer tissues than in normal tissues. Subgroup analysis further confirmed that ABL1 mRNA was highly expressed in HCC. In addition, we used the GEPIA database to evaluate the prognostic value of ABL1 in HCC. We found that high ABL1 expression was significantly associated with adverse OS and DFS. Multivariate Cox regression analysis further showed that the ABL1 expression level was an independent risk factor for liver cancer prognosis.

Coexpressed genes act synergistically in strictly regulated biological processes, and thus they can provide alternative pathways to sidestep barriers, providing an adaptive evolution advantage [24]. We performed enrichment analysis of ABL1-related genes. GO analysis revealed some functional terms related to $A B L 1$, such as the histone modification $(P=2.48 e-09)$, the ATP metabolic process $(P=2.27 e-05)$, and the Wnt signaling pathway $(P=0.035)$ [25-27]. Functional enrichment analysis showed that ABL1 


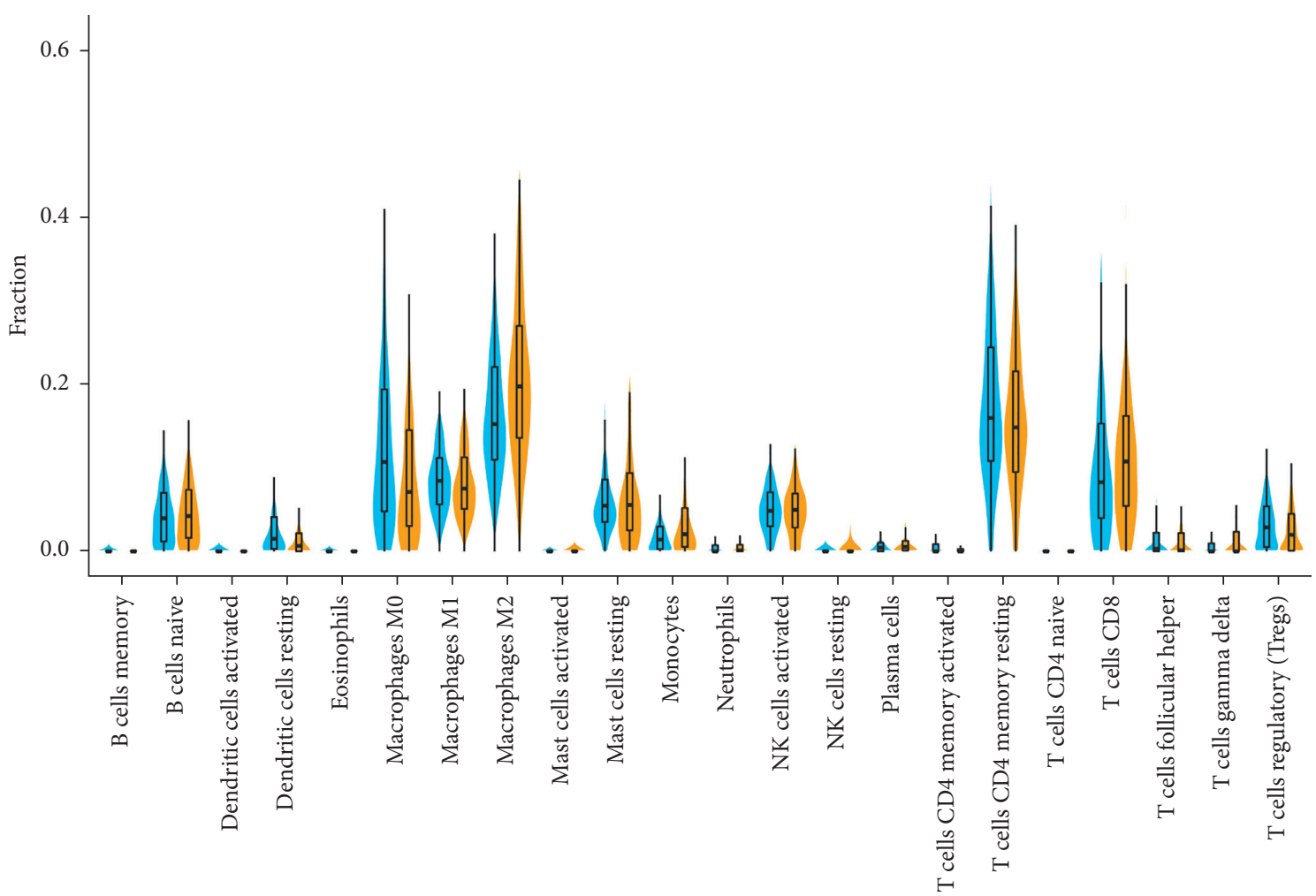

FIgURE 6: The ratios of 22 immune cell subpopulations are analyzed via CIBERSORT. Yellow represents high ABL1 expression group. Blue represents low ABL1 expression group.

was associated with Huntington's disease $(P=1.12 e-08)$, amyotrophic lateral sclerosis $(P=3.0 e-07)$, Alzheimer's disease $(P=3.59 e-07)$, and Parkinson's disease $(P=6.53 e-08)$. Surprisingly, ABL1 was also associated with nonalcoholic fatty liver disease $(P=5.96 e-08)$. In addition, we found pathways and metabolic processes related to tumors, such as oxidative phosphorylation $(P=5.54 e-08), \quad$ inositol phosphate metabolism $(P=0.0035)$, and notch signaling pathway $(P=0.0038)$. These results indicate that ABL1 is involved in a variety of diseases and may play an important role in them. The results also confirmed that ABL1 regulates the progression of $\mathrm{HCC}$ through a complex mechanism, revealing ABL1 as a potential target in HCC treatment.

HCC is a typical inflammatory-related tumor. Its tumor microenvironment includes a large number of immune cells, inflammatory factors, and extracellular matrix, forming a complex immune microenvironment. Immune cells in the tumor microenvironment of HCC mainly include tumorinfiltrating lymphocytes (TILs), tumor-associated macrophages (TAMs), tumor-associated neutrophils (TANs), myeloid-derived suppressor cells (MDSCs), and dendritic cells (DCs). TILs are composed of regulatory $\mathrm{T}$ cells, cytotoxic T lymphocytes, B cells, and NK cells in HCC. Regulatory $\mathrm{T}$ cells are a subset of CD4 $+\mathrm{T}$ cells, a group of lymphocytes with a high degree of immunosuppression, which can achieve immunosuppressive effects by inhibiting CD8 + T cells [28] Studies displayed that regulatory $\mathrm{T}$ cells increased significantly in HCC and were related to tumor size, invasiveness, and prognosis $[29,30]$. Lee et al. found that CD4 + CD $25+$ Treg infiltrated in HCC could effectively inhibit the immune response of dendritic cell [31]. CD8 + T cells are the main cytotoxic T lymphocytes that play an antitumor effect in HCC. Our previous study found that IL-21 produced by $\mathrm{CD} 8+\mathrm{T}$ cells in HCC induced the differentiation of B cells into plasma cells, which stimulated humoral immunity and was associated with favorable prognosis [32]. In addition, the expression of Fas/FasL on CD8 + T cells was positively correlated with the antitumor immunity of liver cancer [33]. $\mathrm{B}$ cells can directly present tumor-related antigens to CD4 + T and CD8 + T cells to exert antitumor immunity or directly kill tumor cells. CD20 (+) B cells in the tumor microenvironment can produce IFN- $\gamma$, interleukin $12 \mathrm{p} 40$, granzyme $\mathrm{B}$, and TRAIL and acted in cooperation with CD8 $(+)$ T cells to promote tumor immunity and predict good prognosis in HCC [34]. However, a study found that PD-1 (hi) B cell infiltration in HCC could induce tumor immune tolerance and lead to poor prognosis [35]. NK cells are an important component of innate immunity, and their defects or inhibition of function can significantly affect the prognosis of HCC patients. Recently, some scholars have found that CD49a + NK cells, a subgroup of NK cells, highly expressing immune checkpoints PD-1 and TIGIT, exert immunosuppressive effects to promote poor prognosis in patients with HCC [36]. TAMs can be divided into M1 type and M2 type. M2 type macrophages can secrete a variety of cytokines and rely on a variety of ways to promote liver cancer invasion and metastasis [37]. However, M1 type can exert effective antitumor effects and inhibit the progression of liver cancer [38]. TANs also are multifaceted. On the one hand, it can recruit 


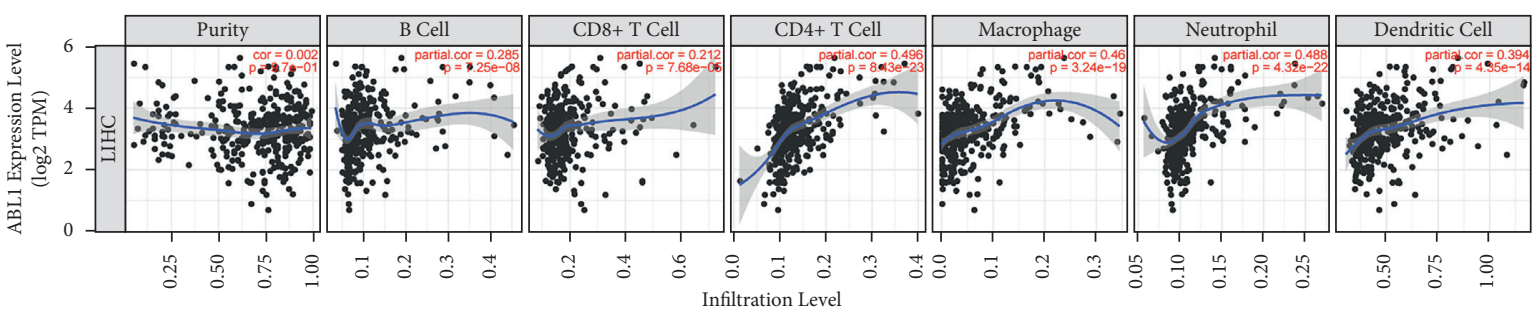

(a)

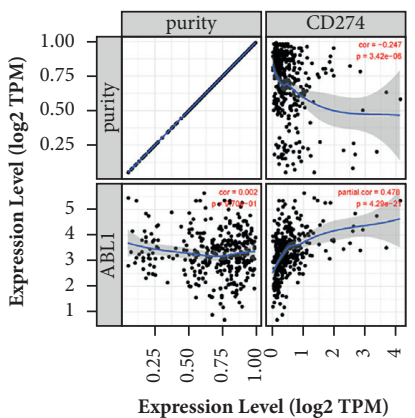

(b)

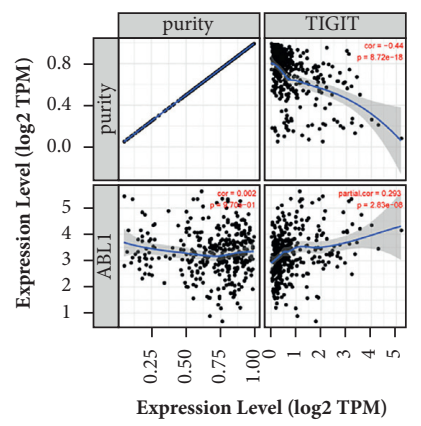

(d)

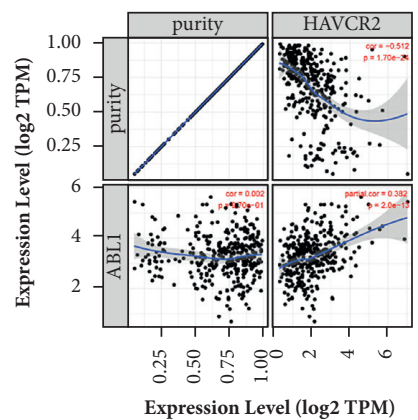

(c)

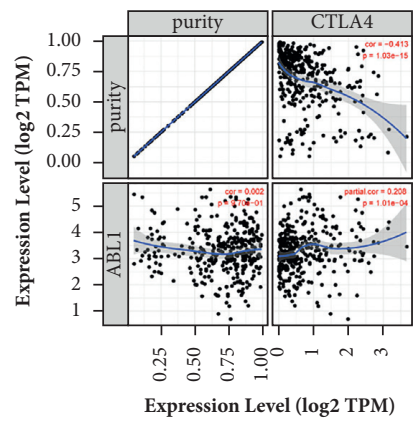

(e)

FIgURE 7: ABL1 expression affects immune cell infiltration in HCC. (a) The relationship between ABL1 expression and immune infiltrating cells was analyzed by TIMER. (b) ABL1 expression is positively correlated with PD-L1, TIGIT, TIM3, and CTLA4 expression.

regulatory T cells and TAMs to promote liver cancer invasion and metastasis [39]. On the other hand, it can directly kill liver cancer cells and inhibit tumor progression [39]. MDSCs are a group of immature myeloid cells with strong immunosuppressive activity and can inhibit antitumor immunity from different ways. Studies revealed that targeting MDSC in HCC could enhance the antitumor effect of immune checkpoint inhibitors [40]. DCs are the most important antigen-presenting cells in the human body, which stimulate adaptive immune responses by presenting antigens to other immune cells. DCs can simultaneously regulate immune response and immune tolerance and play an important role in regulatory immunity. A report revealed that DCs changed from the early state of immune activation to the state of immunosuppression during the progression of liver cancer [41]. Another study showed that plasmacytoid dendritic cells infiltrated in HCC were a risk factor for poor prognosis [42]. They may induce the production of a variety of regulatory cells and inhibit the function of cytotoxic T cells leading to immune escape. We used CIBERSORT to analyze the tumor immune microenvironment of HCC. We found that ABL1 expression was significantly correlated with increased infiltration of B cells, dendritic cells, macrophages, CD4+ cells, and CD8+ cells. We further verified these results with TIMER and found that high ABL1 expression was positively correlated with B cells $(r=0.285, \quad P=7.25 e-08), \quad$ CD $\quad$ cells $\quad(r=0.212$, $P=7.68 e-06), \quad$ CD $4+$ cells $(r=0.496, \quad P=8.43 e-23)$, macrophages $\quad(r=0.46, \quad P=3.24 e-19)$, neutrophils $(r=0.488, P=4.32 e-22)$, and dendritic cells $(r=0.394$, $P=4.35 e-14)$ in HCC. Among these immune cells, CD4+ cells were most strongly associated with ABL1 expression. The findings indicate that ABL1 has an important effect on immune infiltrating cells in HCC.

Immune checkpoints have been proven to be effective targets for the treatment of tumors. Studies have shown that the expression of immune checkpoints such as PD-1L, TIM3, TIGIT, and CTLA4 can affect tumor progression and thus change patient prognosis [43-46]. Immunosuppressants based on immune checkpoints have been effectively used in clinical practice. In the current study, we found that ABL1 expression was positively correlated with PD-1L, TIM3, TIGIT, and CTLA4 expression. Interestingly, we also found that high expression of ABL1 was positively correlated with the expression of markers of these immune checkpoints, 
TABLE 3: Spearman correlation analysis between ABL1 expression and markers of immune cells in HCC.

\begin{tabular}{|c|c|c|c|}
\hline Terms & Markers & $\mathrm{R}$ & $P$ value \\
\hline \multirow{14}{*}{ T cell exhaustion } & PDCD1 (PD-1) & 0.2 & $* * *$ \\
\hline & CTLA4 & 0.111 & $*$ \\
\hline & LAG3 & 0.063 & 0.198 \\
\hline & HAVCR2 (TIM3) & 0.281 & $* * *$ \\
\hline & GZMB & 0.086 & 0.078 \\
\hline & BTLA & 0.087 & 0.074 \\
\hline & CD244 (SLAMF4) & 0.041 & 0.403 \\
\hline & $\mathrm{CD} 274(\mathrm{PD}-\mathrm{L} 1)$ & 0.201 & $* * *$ \\
\hline & CD96 & 0.201 & $* * *$ \\
\hline & IDO1 & 0.1 & $*$ \\
\hline & $\mathrm{KDR}$ & 0.162 & $* * *$ \\
\hline & PDCD1LG2 (PD-L2) & 0.197 & $* * *$ \\
\hline & TGFBR1 & 0.498 & $* * *$ \\
\hline & TIGIT & 0.145 & $* *$ \\
\hline \multirow{4}{*}{ T cell (general) } & CD3E & 0.145 & $* *$ \\
\hline & CD3G & 0.197 & $* * *$ \\
\hline & CD28 & 0.181 & $* * *$ \\
\hline & $\mathrm{CD} 2$ & 0.137 & $* *$ \\
\hline \multirow{2}{*}{$\mathrm{CD} 8+\mathrm{T}$ cells } & CD8A & 0.144 & $* *$ \\
\hline & $\mathrm{CD} 8 \mathrm{~B}$ & 0.098 & ${ }^{*}$ \\
\hline \multirow{3}{*}{$\mathrm{CD} 4+\mathrm{T}$ cells } & $\mathrm{CD} 4$ & 0.059 & 0.228 \\
\hline & CD40LG (CD40L) & 0.143 & $* *$ \\
\hline & CXCR4 & 0.306 & $* * *$ \\
\hline \multirow{4}{*}{ Th1 cells } & TBX21 & 0.093 & 0.055 \\
\hline & STAT4 & 0.242 & $* * *$ \\
\hline & STAT1 & 0.211 & $* * *$ \\
\hline & IFNG & 0.082 & 0.093 \\
\hline \multirow{2}{*}{ Th2 cells } & STAT6 & 0.34 & $* * *$ \\
\hline & STAT5A & 0.448 & $* * *$ \\
\hline \multirow{2}{*}{ Tfh cells } & BCL6 & 0.306 & $* * *$ \\
\hline & IL-21 & 0.092 & 0.058 \\
\hline \multirow{2}{*}{ Th17 cells } & STAT3 & 0.306 & $* * *$ \\
\hline & IL17A & 0.092 & 0.058 \\
\hline \multirow{4}{*}{ Treg } & FOXP3 & -0.035 & 0.473 \\
\hline & STAT5B & 0.375 & $* * *$ \\
\hline & TGFB1 & 0.342 & $* * *$ \\
\hline & IL2RA (CD25) & 0.271 & $* * *$ \\
\hline \multirow{2}{*}{ B cell } & CD19 & 0.072 & 0.141 \\
\hline & CD79A & 0.058 & 0.235 \\
\hline \multirow{2}{*}{ Monocyte } & CD86 (B7-2) & 0.251 & $* * *$ \\
\hline & CSF1R & 0.281 & $* * *$ \\
\hline \multirow{3}{*}{ TAM } & CCL2 & 0.176 & $* * *$ \\
\hline & CD68 & 0.014 & 0.777 \\
\hline & IL10 & 0.258 & $* * *$ \\
\hline \multirow{2}{*}{ M1 macrophage } & IRF5 & 0.316 & $* * *$ \\
\hline & PTGS2 & 0.142 & ${ }^{* *}$ \\
\hline \multirow{3}{*}{ M2 macrophage } & CD163 & 0.09 & 0.063 \\
\hline & VSIG4 & 0.164 & $* * *$ \\
\hline & MS4A4A & 0.219 & $* * *$ \\
\hline \multirow{3}{*}{ Neutrophils } & CEACAM8 & 0.039 & 0.428 \\
\hline & ITGAM & 0.363 & $* * *$ \\
\hline & CCR7 & 0.088 & 0.071 \\
\hline
\end{tabular}


TABLE 3: Continued.

\begin{tabular}{|c|c|c|c|}
\hline Terms & Markers & $\mathrm{R}$ & $P$ value \\
\hline \multirow{8}{*}{ Natural killer cell } & FCGR3A (CD16) & 0.164 & $* * *$ \\
\hline & NCAM1 (CD56) & 0.211 & $* * *$ \\
\hline & KIR2DL1 & 0.067 & 0.167 \\
\hline & KIR2DL3 & 0.157 & $* *$ \\
\hline & KIR2DL4 & 0.171 & $* * *$ \\
\hline & KIR3DL1 & 0.104 & $*$ \\
\hline & KIR3DL2 & 0.13 & $* *$ \\
\hline & KIR2DS4 & 0.01 & 0.844 \\
\hline \multirow{5}{*}{ Dendritic cell } & HLA-DRA & 0.148 & $* *$ \\
\hline & HLA-DPA1 & 0.144 & $* *$ \\
\hline & $\mathrm{CD} 1 \mathrm{C}$ & 0.23 & $* * *$ \\
\hline & NRP1 & 0.53 & $* * *$ \\
\hline & ITGAX & 0.261 & $* * *$ \\
\hline
\end{tabular}

TAM, tumor-associated macrophages; Tfh cells, T follicular helper cells; Treg, T regulatory cells. ${ }^{*} P<0.05,{ }^{* *} P<0.01$, and ${ }^{* * *} P<0.001$.

such as STAT1, STAT3, STAT4, STAT6, STAT5A, and BCL6. In addition, we also observed that ABL1 expression was significantly correlated with monocytes, tumor-associated macrophages, M1/M2 macrophages, NK cells, and dendritic cells. These results show that ABL1 can regulate immune cell infiltration and affect the progression of HCC.

\section{Conclusion}

Our results demonstrated that high ABL1 expression is associated with unfavorable prognosis in HCC. The high ABL1 expression significantly influences the immune cell infiltration and immune checkpoint expression in the tumor microenvironment in HCC. We hypothesize that specific molecular targeting ABL1 expression could affect immune cell infiltration in the tumor microenvironment and improve the prognosis of patients with HCC. Targeting ABL1 expression may effectively strengthen the effectiveness of other immune checkpoint inhibitors in HCC. ABL1 may be a promising prognostic biomarker and therapeutic target for HCC patients. Our research provides a basis for the role of ABL1 in $\mathrm{HCC}$, and further research is strongly recommended.

\section{Abbreviations}

HCC: Hepatocellular carcinoma

ABL1: Abelson tyrosine-protein kinase 1

OS: Overall survival

DFS: Disease-free survival

GEPIA: Gene Expression Profiling Interactive Analysis

KEGG: Kyoto Encyclopedia of Genes and Genomes

TCGA: The Cancer Genome Atlas

TILs: Tumor-infiltrating lymphocytes

TAMs: Tumor-associated macrophages

TANs: Tumor-associated neutrophils

MDSCs: Myeloid-derived suppressor cells

DCs: Dendritic cells.

\section{Data Availability}

All data can be obtained from the first author or corresponding author.

\section{Disclosure}

The funders had no role in study design, data collection and analysis, decision to publish, or preparation of the manuscript.

\section{Conflicts of Interest}

The authors declare that they have no conflicts of interest.

\section{Authors' Contributions}

Guarantors of the article: Linsen Ye and Xiaofeng Yuan. Linsen Ye and Xiaofeng Yuan contributed to the study inception and design. Rongqiang Liu, Weihao Kong, and ZeKun Jiang equally analyzed the data and wrote the manuscript. Shiyang Zheng contributed to the study design and study supervision. All authors approved the final version of the manuscript. Rongqiang Liu, Weihao Kong, and ZeKun Jiang contributed equally to this work.

\section{Acknowledgments}

This work was supported by grants from the Guangdong Basic and Applied Basic Research Foundation (2019A1515110654), Fundamental Research Funds for the Central Universities (20ykpy38), and China Postdoctoral Science Foundation (2019TQ0369 and 2020M672987).

\section{References}

[1] H. B. El-Serag, "Hepatocellular carcinoma," New England Journal of Medicine, vol. 365, no. 12, pp. 1118-1127, 2011.

[2] L. A. Torre, F. Bray, R. L. Siegel, J. Ferlay, J. Lortet-Tieulent, and A. Jemal, "Global cancer statistics, 2012," CA: A Cancer Journal for Clinicians, vol. 65, no. 2, pp. 87-108, 2015.

[3] J. L. Petrick, S. P. Kelly, S. F. Altekruse, K. A. McGlynn, and P. S. Rosenberg, "Future of hepatocellular carcinoma incidence in the United States forecast through 2030," Journal of Clinical Oncology, vol. 34, no. 15, pp. 1787-1794, 2016.

[4] J. Hartke, M. Johnson, and M. Ghabril, "The diagnosis and treatment of hepatocellular carcinoma," Seminars in Diagnostic Pathology, vol. 34, no. 2, pp. 153-159, 2017. 
[5] T. Taki, N. Shibuya, M. Taniwaki et al., "ABI-1, a human homolog to mouse abl-interactor 1 , fuses theMLL gene in acute myeloid leukemia with $t(10 ; 11)(p 11.2 ; q 23)$, , Blood, vol. 92, no. 4, pp. 1125-1130, 1998.

[6] C. Morerio, C. Rosanda, A. Rapella, C. Micalizzi, and C. Panarello, "Is $t(10 ; 11)(p 11.2 ; q 23)$ involving MLL and ABI-1 genes associated with congenital acute monocytic leukemia?" Cancer Genetics and Cytogenetics, vol. 139, no. 1, pp. 57-59, 2002.

[7] E. K. Greuber, P. Smith-Pearson, J. Wang, and A. M. Pendergast, "Role of ABL family kinases in cancer: from leukaemia to solid tumours," Nature Reviews Cancer, vol. 13, no. 8, pp. 559-571, 2013.

[8] C. Wang, R. Navab, V. Iakovlev et al., "Abelson interactor protein-1 positively regulates breast cancer cell proliferation, migration, and invasion," Molecular Cancer Research, vol. 5, no. 10, pp. 1031-1039, 2007.

[9] V. Jenei, T. Andersson, J. Jakus, and K. Dib, “E3B1, a human homologue of the mouse gene product Abi-1, sensitizes activation of Rap1 in response to epidermal growth factor," Experimental Cell Research, vol. 310, no. 2, pp. 463-473, 2005.

[10] M. Cui, W. Yu, J. Dong, J. Chen, X. Zhang, and Y. Liu, "Downregulation of ABI1 expression affects the progression and prognosis of human gastric carcinoma," Medical Oncology, vol. 27, no. 3, pp. 632-639, 2010.

[11] C. Wang, D. Tran-Thanh, J. C. Moreno et al., "Expression of $\mathrm{Abl}$ interactor 1 and its prognostic significance in breast cancer: a tissue-array-based investigation," Breast Cancer Research and Treatment, vol. 129, no. 2, pp. 373-386, 2011.

[12] L. Chitsike, X. Ding, and P. Breslin, "ABL1 is overexpressed and activated in hepatocellular carcinoma," Journal of Cancer and Tumor, vol. 6, no. 8, 2017.

[13] F. Wang, W. Hou, L. Chitsike et al., "ABL1, overexpressed in hepatocellular carcinomas, regulates expression of NOTCH1 and promotes development of liver tumors in mice," Gastroenterology, vol. 159, no. 1, pp. 289-305, 2020.

[14] J. C. Mossane and F. Tacke, "Role of lymphocytes in liver cancer," OncoImmunology, vol. 2, no. 11, Article ID e26468, 2013.

[15] A. Blum, P. Wang, and J. C. Zenklusen, "SnapShot: TCGAanalyzed tumors," Cell, vol. 173, no. 2, p. 530, 2018.

[16] Z. Tang, C. Li, B. Kang et al., "GEPIA: a web server for cancer and normal gene expression profiling and interactive analyses," Nucleic Acids Research, vol. 45, no. W1, pp. W98-W102, 2017.

[17] D. S. Chandrashekar, B. Bashel, S. A. H. Balasubramanya et al., "UALCAN: a portal for facilitating tumor subgroup gene expression and survival analyses," Neoplasia, vol. 19, no. 8, pp. 649-658, 2017.

[18] S. V. Vasaikar, P. Straub, J. Wang, and B. Zhang, "LinkedOmics: analyzing multi-omics data within and across 32 cancer types," Nucleic Acids Research, vol. 46, no. D1, pp. D956-D963, 2018.

[19] T. Li, J. Fan, B. Wang et al., "TIMER: a web server for comprehensive analysis of tumor-infiltrating immune cells," Cancer Research, vol. 77, no. 21, pp. e108-e110, 2017.

[20] A. M. Newman, C. L. Liu, M. R. Green et al., "Robust enumeration of cell subsets from tissue expression profiles," Nature Methods, vol. 12, no. 5, pp. 453-457, 2015.

[21] B. Ru, C. N. Wong, Y. Tong et al., "TISIDB: an integrated repository portal for tumor-immune system interactions," Bioinformatics, vol. 35, no. 20, pp. 4200-4202, 2019.

[22] A. Forner, M. Reig, and J. Bruix, "Hepatocellular carcinoma," The Lancet, vol. 391, no. 10127, pp. 1301-1314, 2018.
[23] M. Tanaka, F. Katayama, H. Kato et al., "Hepatitis B and C virus infection and hepatocellular carcinoma in China: a review of epidemiology and control measures," Journal of Epidemiology, vol. 21, no. 6, pp. 401-416, 2011.

[24] C. Niehrs and N. Pollet, "Synexpression groups in eukaryotes," Nature, vol. 402, no. 6761, pp. 483-487, 1999.

[25] M. Mancini, M. Aluigi, E. Leo et al., "Histone H3 covalent modifications driving response of BCR-ABL1+ cells sensitive and resistant to imatinib to Aurora kinase inhibitor MK0457," British Journal of Haematology, vol. 156, no. 2, pp. 265-268, 2012.

[26] B. J. Lee and N. P. Shah, "Identification and characterization of activating ABL1 $1 \mathrm{~b}$ kinase mutations: impact on sensitivity to ATP-competitive and allosteric ABL1 inhibitors," Leukemia, vol. 31, no. 5, pp. 1096-1107, 2017.

[27] W. Wang, M. Li, S. Ponnusamy et al., “ABL1-dependent OTULIN phosphorylation promotes genotoxic Wnt/ $\beta$-catenin activation to enhance drug resistance in breast cancers," Nature Communications, vol. 11, no. 1, p. 3965, 2020.

[28] E. Cariani and G. Missale, "Immune landscape of hepatocellular carcinoma microenvironment: implications for prognosis and therapeutic applications," Liver International, vol. 39, no. 9, pp. 1608-1621, 2019.

[29] K. W. Huang, K. Jayant, P.-H. Lee et al., "Positive immunomodulation following radiofrequency assisted liver resection in hepatocellular carcinoma," Journal of Clinical Medicine, vol. 8, no. 3, p. 385, 2019.

[30] Y. Huang, F.-M. Wang, T. Wang et al., "Tumor-infiltrating FoxP3+ Tregs and CD8+ T cells affect the prognosis of hepatocellular carcinoma patients," Digestion, vol. 86, no. 4, pp. 329-337, 2012.

[31] W.-C. Lee, T.-J. Wu, H.-S. Chou et al., "The impact of CD4+CD25+ T cells in the tumor microenvironment of hepatocellular carcinoma," Surgery, vol. 151, no. 2, pp. 213-222, 2012.

[32] L. Ye, Y. Li, H. Tang et al., "CD8+CXCR5+T cells infiltrating hepatocellular carcinomas are activated and predictive of a better prognosis," Aging, vol. 11, no. 20, pp. 8879-8891, 2019.

[33] C.-L. Guo, X.-H. Yang, W. Cheng et al., "Expression of fas/ FasL in CD8+T and CD3+Foxp3+Treg cells - relationship with apoptosis of circulating CD8+T cells in hepatocellular carcinoma patients," Asian Pacific Journal of Cancer Prevention, vol. 15, no. 6, pp. 2613-2618, 2014.

[34] J.-Y. Shi, Q. Gao, Z.-C. Wang et al., "Margin-infiltrating $\mathrm{CD} 20+\mathrm{B}$ cells display an atypical memory phenotype and correlate with favorable prognosis in hepatocellular carcinoma," Clinical Cancer Research, vol. 19, no. 21, pp. 5994-6005, 2013.

[35] X. Xiao, X.-M. Lao, M.-M. Chen et al., "PD-1hi identifies a novel regulatory B-cell population in human hepatoma that promotes disease progression," Cancer Discovery, vol. 6, no. 5, pp. 546-559, 2016.

[36] H. Sun, L. Liu, Q. Huang et al., "Accumulation of tumorinfiltrating CD49a+ NK cells correlates with poor prognosis for human hepatocellular carcinoma," Cancer Immunology Research, vol. 7, no. 9, pp. 1535-1546, 2019.

[37] Y. Zhu, J. Yang, D. Xu et al., "Disruption of tumour-associated macrophage trafficking by the osteopontin-induced colonystimulating factor-1 signalling sensitises hepatocellular carcinoma to anti-PD-L1 blockade," Gut, vol. 68, no. 9, pp. 1653-1666, 2019.

[38] J.-Y. Ao, X.-D. Zhu, Z.-T. Chai et al., "Colony-Stimulating factor 1 receptor blockade inhibits tumor growth by altering the polarization of tumor-associated macrophages in 
hepatocellular carcinoma," Molecular Cancer Therapeutics, vol. 16, no. 8, pp. 1544-1554, 2017.

[39] K. Arvanitakis, I. Mitroulis, and G. Germanidis, “Tumorassociated neutrophils in hepatocellular carcinoma pathogenesis, prognosis, and therapy," Cancers, vol. 13, no. 12, Article ID 131, 2021.

[40] J. Zhou, M. Liu, H. Sun et al., "Hepatoma-intrinsic CCRK inhibition diminishes myeloid-derived suppressor cell immunosuppression and enhances immune-checkpoint blockade efficacy," Gut, vol. 67, no. 5, pp. 931-944, 2018.

[41] M. Zhong, C. Zhong, W. Cui et al., "Induction of tolerogenic dendritic cells by activated TGF- $\beta /$ Akt/Smad 2 signaling in RIG-I-deficient stemness-high human liver cancer cells," BMC Cancer, vol. 19, no. 1, p. 439, 2019.

[42] Z.-J. Zhou, H.-Y. Xin, J. Li, Z.-Q. Hu, C.-B. Luo, and S.-L. Zhou, "Intratumoral plasmacytoid dendritic cells as a poor prognostic factor for hepatocellular carcinoma following curative resection," Cancer Immunology, Immunotherapy, vol. 68, no. 8, pp. 1223-1233, 2019.

[43] D. Jiang, Q. Song, H. Wang et al., "Independent prognostic role of PD-L1 expression in patients with esophageal squamous cell carcinoma," Oncotarget, vol. 8, no. 5, pp. 8315-8329, 2017.

[44] W. Du, M. Yang, A. Turner et al., "TIM-3 as a target for cancer immunotherapy and mechanisms of action," International Journal of Molecular Sciences, vol. 18, no. 3, p. 645, 2017.

[45] S. A. Minnie, R. D. Kuns, K. H. Gartlan et al., "Myeloma escape after stem cell transplantation is a consequence of T-cell exhaustion and is prevented by TIGIT blockade," Blood, vol. 132, no. 16, pp. 1675-1688, 2018.

[46] P. Hu, Q. Liu, G. Deng et al., "The prognostic value of cytotoxic T-lymphocyte antigen 4 in cancers: a systematic review and meta-analysis," Scientific Reports, vol. 7, no. 1, Article ID 42913, 2017. 